Ciencia y Sociedad, Vol. 44, No. 4, octubre-diciembre, 2019• ISSN (impreso): 0378-7680 • ISSN (en línea): 2613-8751

DOI: https://doi.org/10.22206/cys.2019.v44i4.pp25-32

\title{
UN CASO DE INJUSTICIA HERMENÉUTICA: LA VIDA DE DONALD SHIRLEY EN LA PELÍCULA GREEN BOOK
}

\section{A case of hermeneutic injustice: The life of Donald Shirley in the movie "Green Book"}

\section{Leonardo Díaz}

Doctor en Filosofía por la Universidad del País Vasco. Director de Investigación de la Facultad de Humanidades de la Universidad Autónoma de Santo Domingo (UASD). Correo-e: leonardodiazsd@gmail.com

ORCID: 0000-0003-3519-0919

Recibido: 4/9/2019 • Aprobado: 7/11/2019

Cómo citar: Díaz, L. (2019). Un caso de injusticia hermenéutica: la vida de Donald Shirley en la película Green Book. Ciencia y Sociedad, 44(4), 25-32. https://doi.org/10.22206/cys.2019.v44i4.pp25-32

Resumen

Desde que la filósofa británica Miranda Fricker creó el concepto de injusticia hermenéutica, se han discutido los límites de la definición original, mostrando otras expresiones de la injusticia hermenéutica. En este artículo, reviso estas expresiones y realizo una reflexión sobre la vida del pianista Donald Shirley en la película Green Book, de Peter Farrely. El propósito de este escrito es presentar la historia de Shirley como un caso de marginación hermenéutica y situarlo dentro de una de las expresiones de injusticia hermenéutica mostrada por Mason y Pérez.

Palabras clave: injusticia hermenéutica; marginación hermenéutica; concepto; experiencia; recursos interpretativos.

\section{Abstract}

Since the British philosopher Miranda Fricker created the concept of hermeneutic injustice, the limits of the original definition have been discussed showing other expressions of hermeneutic injustice. In this article, I review these manifestations and make a reflection on the life of pianist Donald Shirley in Peter Farrely's Green Book movie. The purpose of this paper is to present Shirley's story as a case of hermeneutical marginalization and place it within one of the different expressions of hermeneutic injustice.

Keywords: Injustice hermeneutics; hermeneutical marginalization; concept; experience; interpretative resources. 


\section{Introducción}

En la historia de la epistemología occidental, ha sido común explicar el proceso del conocimiento partiendo del supuesto de un yo abstracto. Ya sea una conciencia autofundante, como Descartes (1998), o un sujeto lógico como Kant (1992), el proceso del conocimiento se ha concebido como el producto de un sujeto no situacional, incondicionado. Relacionado con este supuesto, se ha obviado el hecho de que el proceso epistémico puede verse afectado por la existencia de unas relaciones de desigualdad que generan un acceso inequitativo al bien del conocimiento.

La obra de la filósofa británica Miranda Fricker toma distancia de ambas deficiencias. Su proyecto es un esfuerzo por comprender el proceso del conocimiento a partir de unos sujetos situados y donde las relaciones de desigualdad social pueden generar experiencias incomprendidas para los agentes implicados, especialmente para los que sufren la inequidad.

Fricker (2007) introdujo el concepto de injusticia epistémica en la reflexión filosófica para referirse al fenómeno de experimentar, como sujeto de conocimiento, las desventajas de encontrarse en una relación de subordinación por prejuicios de identidad (género, etnia).

Asimismo, Fricker diferenció dos formas de injusticia epistémica: la injusticia testimonial, que acontece cuando el grado de credibilidad del discurso de un hablante queda disminuido por los prejuicios que un oyente tiene sobre él; y la injusticia hermenéutica, que ocurre cuando una persona se encuentra en una situación desventajosa para comprender sus experiencias sociales producto de una carencia de conceptos que hagan inteligible esas experiencias.

Esto es, un chico de los años ochenta podía sufrir acoso escolar, pero ni él ni sus contemporáneos entendían como tal dicha experiencia. A lo sumo, se entendía como un juego pesado. Nuestras sociedades carecían del concepto de "bullying" y sus implicaciones.

El concepto de Fricker inauguró una línea de investigación fructífera para ampliar nuestra comprensión epistémica y ética de situaciones de marginación social para las personas en función de su género o su etnia. Dicha línea de investigación fue continuada, entre otros, por Maitra (2010), Dotson (2011), Medina (2011), Pérez (2019), Kidd, Medina y Pohlhaus (2017).

Tanto Mason (2011) como Pérez (2019) exploraron otras expresiones de la injusticia hermenéutica no incluidas en la definición original de Fricker. En este sentido, analizar otras expresiones de la injusticia hermenéutica es importante porque nos permite comprender situaciones de marginación y exclusión social no contempladas en la definición original de la filósofa británica.

En la primera parte del artículo, muestro la concepción clásica de Fricker sobre injusticia hermenéutica -alguien que carece del concepto para hacer inteligible su experiencia social— Luego, abordo las expresiones de la injusticia hermenéutica expuestas por Mason (2011) y Pérez (2019). En la segunda parte, reflexiono sobre la vida del pianista Donald Shirley, a partir del análisis de los principales diálogos del filme de Peter Farrely, Green Book, mostrándolo como un caso de marginación hermenéutica y situándolo dentro de una de esas expresiones de Mason (2011) y Pérez (2019) que trascienden la definición original de Fricker (2007).

\section{Injusticia hermenéutica y sus expresiones}

Cuando una persona sufre un daño como sujeto de conocimiento, producto de un prejuicio identitario, nos encontramos ante un caso de injusticia epistémica. Fricker (2017) distingue dos modalidades de la misma: la injusticia testimonial y la injusticia hermenéutica. La primera: 
(...) se produce cuando los prejuicios llevan a un oyente a otorgar a las palabras de un hablante un grado de credibilidad disminuido; la injusticia hermenéutica se produce en una fase anterior, cuando una brecha en los recursos de interpretación colectivos sitúa a alguien en una desventaja injusta en lo relativo a la comprensión de sus experiencias sociales. (Fricker, 2017, pp.17-18).

En las situaciones de injusticia hermenéutica, la persona agraviada intenta comprender sus experiencias sin la ayuda de otro. (Maitra, 2010).

Esta reflexión se enfoca solo en el caso de la injusticia hermenéutica. Fricker la ejemplifica con el relato autobiográfico de Carmita Wood, quien es una empleada que había trabajado durante ocho años en el Departamento de Física Nuclear de la Universidad de Cornell. Durante su estancia en la institución, Wood experimentó sucesivas y constantes experiencias de acoso sexual por parte de un profesor de la institución y desarrolló un cuadro de síntomas físicos que la llevaron a tomar la decisión de pedir un traslado. Cuando este le fue negado, renunció.

Luego, Wood solicitó una asistencia por desempleo. Para conseguirla, debía responder a la pregunta de cuáles habían sido las razones de su renuncia, pero se avergonzaba de describir los detalles y no sabía cómo designar el hecho del que era víctima. Respondió que se había marchado por razones personales. Le fue negada la asistencia.

Wood relató que al decidir tomar acciones legales con un grupo de amigas que habían experimentado otras situaciones de acoso se encontró con que:

El «esto» sobre lo que iban a romper el silencio no tenía nombre (...) Nos referimos en a aquello como "intimidación sexual", "coerción sexual", o "explotación sexual" en el puesto de trabajo. Ninguno de esos nombres nos parecía del todo correcto. Buscábamos algo que recogiera un amplio abanico de conductas persistentes, sutiles y no tan sutiles (...) (Fricker, 2017, pp. 242-243).

Wood sufre lo que Fricker denomina una marginación hermenéutica. Se encuentra en una situación donde es excluida del acceso a significados cuya incomprensión la perjudican desde el punto de vista epistémico, psicológico y social y cuya comprensión se dificulta porque esos significados quedan vedados en la dinámica del sistema social. Mientras Wood y las demás mujeres acosadas:

(...) se desvivían aisladas para encontrar el sentido adecuado de sus diversas experiencias de acoso, el conjunto de la maquinaria de los significados sociales colectivos se engranaba de forma efectiva para mantener esas experiencias apartadas de la vista y en la oscuridad. (Fricker, 2017, p. 246).

Wood adolece de los conceptos apropiados para interpretar su experiencia social y reproduce las interpretaciones sesgadas de los grupos de poder a partir de los prejuicios estructurales de la sociedad.

Desde el punto de vista epistémico, lo que tiene de malo este tipo de marginación hermenéutica es que vuelve estructuralmente prejuicioso el recurso hermenéutico colectivo, ya que tenderá a propiciar interpretaciones sesgadas de las experiencias sociales de ese grupo, porque están insuficientemente influidas por el grupo protagonista y, por tanto, indebidamente influidas por grupos con mayor poder hermenéutico (así por ejemplo, el acoso sexual pasa por flirteo, la violación en el matrimonio como no violación, la depresión posparto como histeria, la reticencia a trabajar horas que dificultan la conciliación de la vida familiar como falta de profesionalidad, etcétera). (Fricker, 2017, p. 249).

Kidd y Carel (2017) señalan que las consecuencias de la marginación hermenéutica surgen porque nuestra habilidad para entender las preocupaciones 
y experiencias de los otros movilizando los recursos hermenéuticos apropiados presupone nuestra habilidad para reconocer y responder a dichas inquietudes y experiencias.

Fricker concentra el problema de la injusticia hermenéutica en la ausencia de recursos interpretativos para comprender las experiencias de la persona que sufre la marginación. No obstante, hay otras situaciones de injusticia hermenéutica no abordadas por Fricker y que han sido detectadas por otros. Por ejemplo, Mason (2011) establece tres situaciones en las que se producen otros tipos de injusticia hermenéutica:

1) Una situación donde los grupos de personas marginados hermenéuticamente son silenciados con respecto a los grupos que poseen los discursos dominantes sin que se les impida comprender su propia experiencia social.

2) Un caso donde los grupos socialmente subordinados disponen de los recursos hermenéuticos para comprender sus experiencias sociales, pero los mismos no son reconocidos ni respetados por los grupos socialmente dominantes o con autoridad epistémica.

3) Una situación de aislamiento epistémico. Se da cuando a una persona o grupo se le clausura el acceso a medios que proporcionan información.

El primer caso puede ser ejemplificado por la situación de Carmita Wood descrita por Fricker.

La segunda situación puede ser ejemplificada con el caso de las culturas precolombinas cuyos discursos no son reconocidos desde la perspectiva del etnocentrismo europeo. Incluye lo que Foucault denominó saberes sometidos: "a toda una serie de saberes que estaban descalificados como saberes no conceptuales, como saberes insuficientemente elaborados: saberes ingenuos, saberes jerárquicamente inferiores, saberes por debajo del nivel del conocimiento o de la cientificidad exigidos". (Foucault, 2002, p. 21)

Pereira (1999) y Dussel (2011 y 2015), se han referido a dicha situación donde los saberes producidos en el determinado tercer mundo son ignorados o considerados carentes de cientificidad o de rigor filosófico.

Una muestra del tercer caso lo constituye la censura estructural tal como la describen Sinova (2006), así como Geoffray y Chaguaceda (2014). En ambos casos, se aíslan a comunidades enteras de la información, la cual es centralizada por el Estado para evitar fisuras o posibles rupturas en el sistema político.

Por su parte, Pérez (2019) distingue cuatro expresiones de la injusticia hermenéutica:

1- Siguiendo a Fricker (2017), señala la posibilidad de una incomprensión de la experiencia social por la ausencia de categorías hermenéuticas.

2- Como Mason (2011), se refiere a la injusticia hermenéutica como producto del rechazo a las categorías construidas por las comunidades para comprender sus experiencias sociales.

3- Incluye la situación donde existe falta de voluntad para comprender los conceptos elaborados por las comunidades epistémicamente subalternas.

4- Y, finalmente, establece que pueden producirse situaciones donde las comunidades creen poder interpretar sus experiencias sociales a partir de los conceptos proporcionados por los grupos que son responsables de su marginación. Pérez y Radi (2018) denominan esta situación como espejismo hermenéutico. 
Pérez (2019) advierte que no debemos percibir las situaciones de injusticia hermenéutica como casos donde una persona en situación de poder ejerce un acto de violencia o dańa a una persona marginada. Retoma el concepto de "ahogamiento epistémico" formulado por Dotson (2011). Tomando como punto de partida el concepto de violencia epistémica enunciado por Spivak (1994), que conlleva silenciar a las comunidades subordinadas, Dotson explora otras formas de silenciamiento incluyendo métodos de acallar contenidos del propio testimonio por parte de los agentes marginados.

En la segunda parte de este artículo abordaré un caso de injustica hermenéutica que desborda la descripción clásica de Fricker. Se da entre dos interlocutores donde uno se encuentra en una posición de poder y se acerca a una de las descripciones de injusticia hermenéutica de Mason (2011) y Pérez (2019). Se trata de la historia del virtuoso del piano Donald Walbridge Shirley (1927-2013), llevada al cine por Peter Farrely Green Book, con el guion de Nick Vallelonga ${ }^{1}$.

\section{El caso de Don Shirley}

A primera vista, Donald Shirley, mejor conocido como Dr. Shirley, parece ser un individuo consciente de su situación en el mundo. Ha recibido una educación privilegiada como músico en Leningrado y ha estudiado psicología en Chicago. No ha sido ajeno a la atmósfera racista del sur de los Estados Unidos de los años sesenta. De hecho, ha aceptado realizar una gira por los estados sureños tratando de modificar la actitud hacia los negros a través de su arte. En este sentido, Shirley es un individuo comprometido y aparentemente lúcido a la hora de interpretar lo que le acontece a él y a su etnia.

1. Los diálogos de Green Book que aparecen en el próximo acápite han sido copiados directamente de la película.
Shirley dispone de categorías para interpretar su experiencia social. A medida que avanzaba la carrera del pianista se desarrollaba en Estados Unidos el movimiento de los derechos civiles que había generado conciencia sobre el problema del racismo y conducido a las leyes de igualdad racial de los años sesenta.

Pero el asunto no es tan simple. Los nuevos discursos de igualdad racial no eran aceptados por segmentos importantes de la sociedad norteamericana, especialmente en el sur de los Estados Unidos. Podríamos catalogar la situación de Shirley dentro de lo que Mason (2011) ejemplifica como un caso de injusticia hermenéutica producto de la falta de reconocimiento y respeto de un grupo socialmente dominante a uno subordinado, en este caso, la población afroamericana. Pero también, puede encajar dentro de lo que Pérez (2019) considera como una situación de rechazo debido a una falta de voluntad para comprender los conceptos elaborados por un grupo sometido, ya que la evidencia científica sobre la irracionalidad del racismo existe desde inicios del siglo xx y era de dominio común para mediados del siglo pasado. Por tanto, la falta de reconocimiento y respeto a la etnia de Shirley es también una indiferencia hacia los datos que muestran dicha falta de reconocimiento y de respeto como un mero prejuicio racial.

El impacto de ese prejuicio es tal que, como ocurre con frecuencia con los individuos que pertenecen a grupos oprimidos, Shirley reproduce los valores de quienes lo marginan. Ha sido educado como un hombre blanco, sus referentes culturales, su forma de vestir y su lenguaje corporal responden a los estereotipos de un norteamericano blanco de las primeras décadas del siglo xx. Quiere modificar el acento y las maneras de su chofer, Tony Vallelonga, para adecuarlos a los prejuicios sociales de los hombres de la clase alta del sur norteamericano. En otras palabras, Shirley quiere modificar los prejuicios hacia los negros, pero no es consciente de que él mismo los reproduce, porque no los ve como 
prejuicios de una etnia y una clase, sino como las formas universales correctas de un hombre educado. Posee tal vez, el peor de los prejuicios que un hombre dominado puede poseer, percibir como universal el prejuicio de un grupo particular dominante.

En el restaurant de Alabama es recibido con pleitesía por el gerente. Sin embargo, cuando intenta cenar en el mismo salón donde él es el protagonista del concierto, se le niega el acceso. El gerente se lo explica:

"Son tradiciones de mucho tiempo. Reglas del club. Sé que lo entiende".

El gerente no comprende la resistencia de Shirley. En un aparte con Vallelonga, trata de que convenza al pianista de que:

"Dígale que no lo estamos insultando personalmente".

La situación de Shirley desborda la mera situación de dos personas donde una oprime a otra. Se trata de una situación estructural.

Shirley llega a reflexionar sobre los aspectos de su experiencia incomprendida por las paradojas de su biografía:

Se siente excluido por los blancos por ser negro, pero también por los negros que le ven diferente.

"Sí. Vivo en un castillo, Tony. ¡Solo! Y los blancos ricos me pagan para tocar el piano... para ellos, porque los hace sentir cultos.

Pero tan pronto como me bajo del escenario vuelvo a ser otro negro para ellos. Porque esa es su verdadera cultura.

Y yo sufro ese desaire solo, porque no soy aceptado por mi propia gente, iporque yo tampoco soy como ellos!
Así que si no soy lo suficientemente negro, y si no soy lo suficientemente blanco, y si no soy lo suficientemente hombre (sentía atracción por los hombres), entonces dime Tony, ¿̇qué soy!?”

"Vivo en un castillo... ¡Solo!" Uno de los principales problemas de Shirley es que intenta enfrentar el problema solo. Como señala Fricker (2017) refiriéndose a la historia del movimiento de los derechos civiles de las mujeres, gran parte del proceso de hacer inteligible los significados de las experiencias opresivas pasó por la interacción entre las mujeres mismas y la capacidad para compartir las experiencias incomprendidas. Shirley carece de esta oportunidad. Vive aislado de los suyos, comenzando por su familia, no tiene amigos, intenta salvar una etnia de la que sin saberlo se siente superior solo a través de su arte.

Shirley puede entender parcialmente su situación opresiva, pero no puede comprenderla del todo, porque sufre de una marginación hermenéutica parcial. Fricker emplea el concepto de marginación hermenéutica para referirse a la situación de un grupo que "sufre de una participación hermenéutica desfavorable en alguna área significativa de la experiencia social" (Fricker, 2017, p. 246). Pero dicha marginación no tiene que ser total. En el caso de Shirley, su condición de individuo formado en universidades de primer nivel y haber alcanzado un doctorado, de codearse con clases educadas y ricas, le concede el acceso a determinadas experiencias significativas ajenas a otros individuos de su etnia que no tuvieron la oportunidad de su educación.

Pero precisamente esta situación vela aún más las marginaciones hermenéuticas que acontecen en otras esferas de la vida cotidiana o en la misma esfera donde se desenvuelve Shirley en otros momentos donde no desempeña su papel de concertista.

El daño no es solo epistémico, sino también psicológico y moral. Volvamos a la situación del diálogo entre Shirley y Tony. 
"Así que si no soy lo suficientemente negro, y si no soy lo suficientemente blanco, y si no soy lo suficientemente hombre (sentía atracción por los hombres), entonces dime Tony, “ïqué soy!?”.

Shirley no solo sufre un problema de comprensión. Su situación le genera un problema de identidad. Su pregunta “¿iqué soy!?” la realiza en un acto de visible desesperación. No es extraño que Shirley se refugie en el alcohol para calmar su crisis. $\mathrm{Ni}$ siquiera su posición privilegiada con respecto a otros afroamericanos le evita la degradación moral expresada en su exclusión de los bienes materiales y culturales. Shirley es víctima de una injusticia hermenéutica sistemática.

Como ha señalado Medina (2011), las injusticias deben analizarse como fenómenos que se desarrollan en la sociedad y en el tiempo, enraizados en modelos de relaciones sociales. El caso de Donald Shirley resulta ilustrativo para comprender cómo la injusticia hermenéutica desborda los casos en que se carece de recursos hermenéuticos para interpretar las experiencias sociales propias y que no es un problema reducible a la conversación del intercambio testimonial entre dos personas.

\section{Conclusiones}

Luego de mostrar el concepto de injusticia hermenéutica concebido por Fricker, he explorado otras modalidades de injusticia hermenéutica a partir de las obras de Mason y de Pérez.

La historia de Donald Shirley es un ejemplo de una de esas mencionadas modalidades que pueden consistir en la falta de reconocimiento de las categorías hermenéuticas con que un determinado grupo comprende sus experiencias sociales, sin excluir también la situación donde el grupo dominante carece de la voluntad para comprender dichas categorías.

En otras palabras, el problema de la injusticia hermenéutica puede producirse, aunque el individuo afectado posea los recursos conceptuales necesarios para hacer inteligible sus experiencias sociales.

Al mismo tiempo, el problema de la injusticia hermenéutica desborda la cuestión de una relación desigual entre dos individuos, pues se produce en el marco de unas relaciones sociales estructurales.

\section{Referencias}

Descartes, R. (1998). Discurso del método. México, Estados Unidos Mexicanos: Ed. Losada.

Dotson, K. (2011). Tracking Epistemic Violence, Tracking Practices of Silencing. Hypatia, 26(2), pp. 236-257.

Dussel, E. (2011). Filosofía de la liberación. México, Estados Unidos Mexicanos: Fondo de Cultura Económica.

Dussel, E. (2015). Filosofías del sur. Descolonización $y$ transmodernidad. México, Estados Unidos Mexicanos: Ediciones Akal.

Foucault, M. (2002). Defender la sociedad. México, Estados Unidos Mexicanos: Fondo de Cultura Económica.

Fricker, M. (2007). Epistemic Injustice: Power and the Ethic of Knowing. New York, Estados Unidos: Oxford University Press.

Fricker, M. (2017). Injusticia epistémica. Barcelona, España: Ed. Herder.

Geoffray, M.L. y Chaguaceda, A. (2014). Medios de comunicación y cambios en la política de información en Cuba desde 1959. Temas de comunicación, 29, pp. 171-196.

Kant, I. (1992). Crítica de la razón pura. Buenos Aires, Argentina: Ed. Losada.

Kidd, I. and Carel, H. (2017). Epistemic Injustice and Illness. Journal of Applied Philosophy, 34(2), pp. 173-190.

Kidd, I., Medina, J. and Pohlhaus, G. (2017). Handbook of Epistemic Injustice. New York, Estados Unidos: Routledge Handbooks.

Maitra, I. (2010). The Nature of Epistemic Injustice. Philosophical Books, 51(4), pp.195-211. 
Mason, R. (2011). Two Kinds of Unknowing. Hypatia, 26, pp. 299-307

Medina, J. (2011). The relevance of credibility excess in a proportional view of epistemic injustice: differential epistemic authority and the social imaginary. Social Epistemology, 25(1), pp. 15-35

Pereira, A. (1999). La epistemología frente al reconocimiento del conocimiento en las ciencias naturales. En J. Maerk y M. Cabriolé (Coords.) ¿Existe una epistemología latinoamericana? (pp. 63-75). México, Estados Unidos Mexicanos: Plaza y Valdés, S.A.
Pérez, M. (2019). Violencia epistémica: Reflexiones entre lo invisible y lo ignorable. Revista de Estudios y Políticas de Género, 1, pp. 81-98. Pérez, M. y Radi, B. (2018). El concepto de violencia de género como "espejismo hermenéutico". Igualdad, autonomia personal y derechos sociales, 8, pp. 69-88.

Sinova, J. (2006). La censura de prensa durante el franquismo. Barcelona, Espańa: Random House.

Spivak, G.Ch. (1994): Can the Subaltern Speak? En Williams, P. and Chrisman, L. Colonial Discourse and Post-Colonial Theory (pp. 66111). New York, Estados Unidos: Columbia University Press.

\section{Datos de filiación}

Leonardo Díaz. Doctor en Filosofía por la Universidad del País Vasco. Premio Nacional de Ensayo Científico (2014). Premio de Ensayo Pedro Francisco Bonó (2012). Miembro de Número de la Academia de Ciencias de la República Dominicana donde coordina la Comisión de Filosofía y Epistemología. Director de Investigación de la Facultad de Humanidades de la Universidad Autónoma de Santo Domingo (UASD). Dirigió la Escuela de Filosofía de la UASD (2014-2018). Ha sido integrante de la Comisión de Alto Nivel para la Difusión de la Filosofía de la UNESCO. Autor de: Reflexiones filosóficas. Artículos de ética, politica y filosofia. (2018); Las tensiones de Thomas Kuhn: una perspectiva critica para los estudios sociales y culturales de la ciencia (2014); La filosofía y los espacios de la libertad (2012), así como de diversos artículos publicados en revistas especializadas nacionales e internacionales. 